\title{
Soil reinforcement with geosynthetics
}

\author{
Mohammed Mustapha BESSAIM ${ }^{1,3}$, Aicha BESSAIM ${ }^{2}$, Hanifi MISSOUM ${ }^{1,3}$, Karim BENDANI ${ }^{1,3}$ \\ ${ }^{1}$ University Abdelhamid Ibn Badis, Department of Civil Engineering, Mostaganem, Algeria \\ ${ }^{2}$ University Mustapha Stambouli, Department of Civil Engineering, Mascara, Algeria \\ ${ }^{3}$ Laboratory Construction, Transport and Environmental Protection
}

\begin{abstract}
The proportionality of existence of land with good bearing to erect any building or building is very small, to remedy this deficiency it is necessary to resort to techniques of reinforcement of the soils which can constitute a very important development. Among these methods of remediation, there is reinforcement by the geosynthetics which constitute an effective solution to these constraints. This process tends to stabilize the soil in question with increased load bearing capacity in civil engineering and geotechnical works such as embankments, slopes, embankments and hydraulic structures, with an inestimable gain in time, economy and durability while preserving the natural and environmental aspect.
\end{abstract}

Keywords: Soil, Geosynthetiques, Reinforcement, Improvement,

\section{Introduction}

Avec le développement et l'accroissement urbains et industriels, des terrains avec de bonnes conditions de construction sont moindres. Ces sols présentent des propriétés inadéquates pour une construction sûre et durable. Parmi ces sols, on peut distinguer les sols fins expansifs et de très grande compressibilité à l'instar des limons et les sols argileux. De même, les sols grenus et les sables fins lâches sont également des matériaux qui nécessitent une étude minutieuse avant de procéder à tous travaux de constructions. L'utilisation et la mauvaise étude de ces derniers engendrent des dégâts et des défauts irrémédiables entrainant usuellement la ruine de l'ouvrage en question. A cet effet, les chercheurs ont pensés à mettre en place des modalités de traitement, parmi lesquelles on peut citer le renforcement par les géosynthétiques dont le but d'exploiter un maximum de tels sols. Ces techniques constituent des avantages inestimables et permettent de résoudre les problèmes liés à l'instabilité ainsi qu'aux différents désordres rencontrés dans le domaine de génie civil car elles permettent d'une part d'améliorer les caractéristiques de l'ouvrage et d'autre part elles de préserver l'aspect esthétique et cela avec un coût réduit [1,2]. Ce processus a suscité l'intérêt de différents chercheurs qui ont étudiés la performance de ce procédé vis-à-vis la portance des fondations renforcée par ces matériaux [3-6] ou même pour le renforcement des pentes $[7,8]$. Après leurs applications avec succès sur le terrain, ces produits ont vite immergés le domaine de génie civil notamment le domaine de la géotechnique car ils ont pu satisfaire aux différents recommandations exigés dans les divers projets. Par ailleurs, les géosynthétiques fournissent une variété d'avantages tels qu'un gain dans la durée de vie des constructions, une amélioration des caractéristiques géotechniques du sol tout en augmentant les caractéristiques mécaniques, la possibilité de construction dans des milieux défavorables, sans oublier l'aspect économique car ils permettent de limiter les coûts de transport et d'installation en termes de structure et d'emprise au sol. En outre, le traitement des sols avec les géosynthétiques permet de préserver l'aspect écologique et environnemental de l'ouvrage.

Dans le présent article, nous allons entamés le renforcement des sols par les géosynthétiques tout en abordant également les différentes applications de ces premiers. De même, nous allons cités les différents types de géosynthétiques, leurs domaines d'utilisation notamment dans le domaine de la géotechnique. 


\section{Les différents géosynthétique}

La forte demande de renforcement par les géosynthétiques ne cesse de s'amplifier et cela en raison des différents types de ces matériaux qui permettent une réalisation rapide, facile et moins coûteuse et cela dépend des différents contextes de l'édifice et cela c'est à cause dans la variété de choix et types qu'offre ces produits. Ils existent différent type de géosynthétiques tels que les géotextiles, les géogrilles, les géomembranes, les géofilets et les géocomposites [9].

Le choix du type de géosynthétique approprié, nécessitent une compréhension de chaque type qui correspond à une fonction principale pour une application spécifique considérée comme le montre le tableau suivant :

Tableau 1. Les types de géosynthétiques en fonction de leurs applicabilités [10].

\begin{tabular}{|c|c|c|c|}
\hline \multirow{2}{*}{ Type } & \multicolumn{3}{|c|}{ Différentes fonctions } \\
\cline { 2 - 4 } Géotextile & $\begin{array}{c}\text { Séparaion } \\
\text { Fonction } \\
\text { principale } \\
\text { ou Fonction } \\
\text { secondaire }\end{array}$ & $\begin{array}{c}\text { Fonction } \\
\text { principale ou } \\
\text { Fonction } \\
\text { secondaire }\end{array}$ & $\begin{array}{c}\text { Fonction } \\
\text { principale } \\
\text { ou } \\
\text { Fonction } \\
\text { secondaire }\end{array}$ \\
\hline Géogrilles & $\begin{array}{c}\text { Fonction } \\
\text { secondaire }\end{array}$ & $\begin{array}{c}\text { Fonction } \\
\text { principale }\end{array}$ & $\begin{array}{c}\text { Non } \\
\text { applicable }\end{array}$ \\
\hline Géofilets & $\begin{array}{c}\text { Fonction } \\
\text { secondaire }\end{array}$ & $\begin{array}{c}\text { Non } \\
\text { applicable }\end{array}$ & $\begin{array}{c}\text { Non } \\
\text { applicable }\end{array}$ \\
\hline Géomembranes & $\begin{array}{c}\text { Fonction } \\
\text { secondaire }\end{array}$ & $\begin{array}{c}\text { Non } \\
\text { applicable }\end{array}$ & $\begin{array}{c}\text { Non } \\
\text { applicable }\end{array}$ \\
\hline Géocomposites & $\begin{array}{c}\text { Fonction } \\
\text { principale } \\
\text { ou Fonction } \\
\text { secondaire }\end{array}$ & $\begin{array}{c}\text { Fonction } \\
\text { principale ou } \\
\text { Fonction } \\
\text { secondaire }\end{array}$ & $\begin{array}{c}\text { Fonction } \\
\text { principale } \\
\text { ou } \\
\text { Fonction } \\
\text { secondaire }\end{array}$ \\
\hline
\end{tabular}

\begin{tabular}{|c|c|c|}
\hline \multirow{2}{*}{ Type } & \multicolumn{2}{|c|}{ Différentes fonctions } \\
\hline & Drainage & Barrière \\
\hline Géotextile & $\begin{array}{c}\text { Fonction principale } \\
\text { ou Fonction } \\
\text { secondaire } \\
\end{array}$ & $\begin{array}{c}\text { Non } \\
\text { applicable }\end{array}$ \\
\hline Géogrilles & $\begin{array}{c}\text { Non } \\
\text { applicable }\end{array}$ & $\begin{array}{c}\text { Non } \\
\text { applicable }\end{array}$ \\
\hline Géofilets & Fonction principale & $\begin{array}{c}\text { Non } \\
\text { applicable }\end{array}$ \\
\hline Géomembranes & $\begin{array}{c}\text { Non } \\
\text { applicable }\end{array}$ & Fonction principale \\
\hline Géocomposites & $\begin{array}{c}\text { Fonction principale } \\
\text { ou Fonction } \\
\text { secondaire }\end{array}$ & $\begin{array}{c}\text { Fonction principale } \\
\text { ou Fonction } \\
\text { secondaire }\end{array}$ \\
\hline
\end{tabular}

\section{Le renforcement des ouvrages par les géosynthétiques}

Les géosynthétiques permettent d'améliorer les caractéristiques du sol en question, car ces premiers possèdent une grande capacité à la traction ce qui est quasi nul dans les sols. Ce type de traitement pourra être opté dans différents contextes tels que les chaussées, les pentes et les murs de soutènement [11,12]. Les différentes applications de renforcement avec les géosynthétiques sont réalisées dans le cas des remblais, les talus ainsi que pour les murs de soutènement comme le montre la figure suivante:

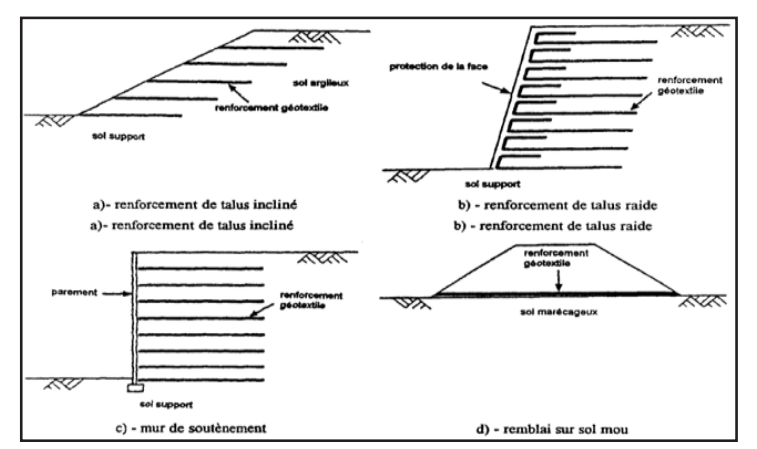

Fig. 1. Divers applications de renforcement des ouvrages avec géosynthétiques [13].

L'exemple de renforcement des pentes avec les géosynthétiques a été abordé par [14]. La stabilité des pentes peut grandement être améliorée avec les géosynthétiques suivant la formule suivante :

$$
F . S=\sum \frac{\text { moments résis } \tan t}{\text { moments moteur }}
$$

$F . S=\frac{\left(\tau_{e} L_{a b}+\tau_{f} L_{b c}\right) R+T_{a} Y}{W X}$

Avec :

$\tau_{e}$ : La résistance de cisaillement de remblai

La-b : Longueur de l'arc a-b

$\tau_{\mathrm{f}}$ : La résistance au cisaillement de la fondation du sol

$\mathrm{L}_{\mathrm{b}-\mathrm{c}}:$ Longueur de l'arc b-c

$\mathrm{R}$ : rayon depuis le centre critique de rupture.

$\mathrm{T}$ : la force de tension admissible du géotextile

$\mathrm{Y}$ : moment de géotextile

$\mathrm{W}$ : Poids du sol dans la zone de rupture.

$\mathrm{X}$ : moment depuis le centre de gravité vers le centre de rupture. 


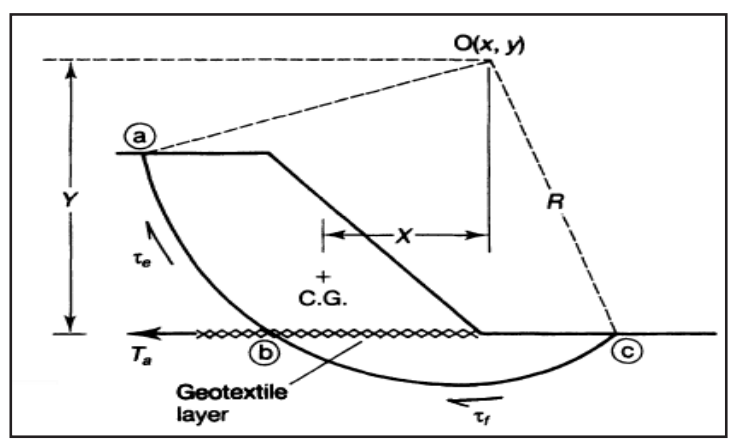

Fig. 2. Configuration générale utilisée pour modifier la stabilité des pentes avec les géosynthétiques [14].

Où il a été remarqué d'après l'équation précédente et depuis cette figure que la résistance du géotextile peut être augmentée si nécessaire pour conduire le facteur de sécurité jusqu'à une valeur acceptable [14]. Il est à connaitre que l'utilisation des géosynthétiques change selon le contexte d'étude prédéfinie. En terme de fonction mécanique, il faut assurer la reprise de forces de traction et de les reporter dans le sol par le biais de forces de cisaillement. Du point de vue hydraulique, il s'agit d'assurer une perméabilité suffisante afin d'éviter une retenue d'eau au-dessus du géotextile [13]. Les propriétés déterminantes des géosynthétiques afin assurer leurs rôles de renforcement. Ces derniers sont illustrés dans le tableau suivant:

Tableau 2. Propriétés déterminantes du géotextile pour un rôle de renforcement [13].

\begin{tabular}{|c|c|c|}
\hline $\begin{array}{c}\text { Functions } \\
\text { partielles }\end{array}$ & Proprieties & Parameters caractéristiques \\
\hline $\begin{array}{c}\text { Function } \\
\text { mécanique }\end{array}$ & $\begin{array}{c}\text { Déformation } \\
\text { Fluage } \\
\text { Frottement }\end{array}$ & $\begin{array}{c}\text { Courbe « force-allongement } \\
\text { Augmentation de la } \\
\text { déformation sous force de } \\
\text { traction constante } \\
\varphi_{s g} \text { Angle de frottement } \\
\text { sol-géotextile }\end{array}$ \\
\hline $\begin{array}{c}\text { Fonction } \\
\text { hydraulique }\end{array}$ & $\begin{array}{c}\text { Perméabilité } \\
\text { normale au } \\
\text { plan du } \\
\text { géotextile }\end{array}$ & $\begin{array}{c}\psi \text { Permittivité } \\
k_{n} \text { Perméabilité }\end{array}$ \\
\hline
\end{tabular}

\section{Conclusion}

Le renforcement des sols par les géosynthétiques représente un moyen d'apport inestimable. L'utilisation de ce procédé est connue par son efficacité depuis des décennies. Cette méthode s'est largement développée pour une vaste gamme d'applications dans divers domaines de géotechniques à l'instar du domaine mécanique et hydraulique. Cette technique est envisageable dans le cas des sols dont les propriétés sont inadéquates et qui répondent mal aux exigences et aux conditions des ouvrages et structures à supporter, d'où la nécessité d'un traitement au préalable. Ce processus permet un gain de la durée de vie des constructions et cela grâce à une méthode relativement facile à mettre en œuvre à des prix moins élevés vis-à-vis d'autres techniques de traitement. Le renforcement par les géosynthétiques est considéré comme étant une alternative avec des avantages prodigieux qui permettent d'une part d'améliorer les propriétés géotechniques du sol et d'autre part de préserver l'aspect naturel et environnemental.

\section{Références}

1. Leshchinsky, D., Han, J., Geosynthetic reinforced multitiered walls. J. Geotech. Geoenviron. Eng. 130 (12), 1225e1235 (2004).

2. Ruan, X., Leshchinsky, D., Leshchinsky, B.A., Global stability of bilinear reinforced slopes. Transp. Infrastruct. Geotechnol. 2, 34e46 (2015).

3. Yetimoglu, T.,Wu, J.T.H., Saglamer, A., Bearing capacity of rectangular footings on geogridreinforced sand. ASCE J. Geotechnical Eng. 120 (12), 2083e2099 (1994).

4. Dash, S., Sireesh, S., Sitharam, T., Model studies on circular footing supported on geocell reinforced sand underlain by soft clay. Geotext. Geomembranes 21 (4), 197e219 (2003).

5. Boushehrian, J.H., Hataf, N., Experimental and numerical investigation of the bearing capacity of model circular and ring footings on reinforced sand. Geotext. Geomembranes 21 (4), 241e256 (2003).

6. Ghosh, A., Ghosh, A., Bera, A.K., Bearing capacity of square footing on pond ash reinforced with jutegeotextile. Geotext. Geomembranes 23 (2), 144e173 (2005).

7. El Sawwaf, M.A., Strip footing behavior on pile and sheet pile stabilized sand slope. J. Geotech. Geoenvironmental Eng. 131 (6), $705 \mathrm{e} 715$ (2005).

8. El Sawwaf, M.A.,Behavior of strip footing on geogrid-reinforced sand over a soft clay slope. Geotext. Geomembranes 25 (1), 50e60 (2007)..

9. Koerner, R. M. Designing with Geosynthetics, 2nd Edition, Prentice-Hall, Inc., Englewood Cliffs, N.J (1990).

10. Koerner, R. M., A note on geotextile design methods, Geotechnical Fabrics Report, 2, No.2, pp. 28-29 (1984).

11. Hausmann, M. R., Fabric reinforced unpaved road design methods-Parametric studies, Proceedings of the Third International Conference on Geotextiles, Vienna, Austria, IFAI, pp. 19-24 (1986).

12. Yako, M. A. and Christopher, B. R., Polymerically reinforced retaining walls and slopes in North America, Workshop on Applications of Polymeric Reinforcement in Soil Retaining Structures, ed. P. Jarrett, Royal Military College, Kingston, Ontario, Canada, pp.213-226(1987).

13. Sere, A. "Ouvrages renforcés par des géotextiles chargés en tête : Comportement et Dimensionnement". Thèse de Doctorat de l'Ecole Nationale des Ponts et Chaussées, 327 pages (1995).

14. Koerner, R. M., Geosynthetics in geotechnical engineering, Chapter 22, Foundation Engineering Handbook, 2nd ed. Kluwer Academic Publishers, Boston, MA, pp. 796-813(1991). 